\title{
Correlation of Magnetic Resonance Spectroscopy with Histopathology of Brain Biopsies
}

\author{
Ali Bashir Ali Elhaj ${ }^{2,5}$, Mohammed Ahmed Ali Omer, ${ }^{1,2}$ Mogahid M. A Zidan ${ }^{4,5}$, Wadah M. Ali ${ }^{3,4}$, \\ Jihad Hamid ${ }^{4}$ \\ ${ }^{1}$ College of Applied Medical Science-Department of Radiologic Technology- Qassim University, Buraidah, KSA
}

${ }^{2}$ Department of Radiotherapy \& Nuclear Medicine, College of Medical Radiologic Science, Sudan University of Science and Technology, Khartoum, Sudan

${ }^{3}$ Medical Imaging Department, College of Allied Medical Science, Gulf Medical University, Ajman, UAE

${ }^{4}$ Nuclear Medicine Department, College of Radiological Science and Nuclear Medicine, The National Ribat University, Khartoum, Sudan

${ }^{5}$ Al-Ghad International College for Applied Medical Science, Medical Imaging Technology Department, Abha, KSA

\begin{abstract}
The pathology of human tissue can be determined by magnetic resonance spectroscopy (MRS) but it was a controversial field for over 20 years. Now MRS on human biopsies identifies disease processes, neoplastic status, and prognostic variables with high accuracy. The MRS databases were compared with careful and specialized histology,. The MR method is fast, accurate, and robust and for most organs complements routine histopathological diagnosis. Study was performed to evaluate the rule of Magnetic Resonance Spectroscopy in diagnosis of brain tumors compare by histopathology. A total of 38 patients were examined in this study. The data collected from three hospitals in Khartoum state from 2014 to July 2015. The patients were examined with the own department protocol using Magnetic resonance machine. The study founded that $(86.8 \%)$ of patients examined by Spectroscopy have same results compare to the histopathology results. Conclusion: MRS is an accurate, noninvasive diagnostic technique for quantifying brain tumors.
\end{abstract}

Keywords: Brain-tumors, Spectroscopy, Histopathology, Metabolite

\section{Introduction}

As stated The incidence of brain tumors (BTs) increases annually by (De Moor, Janet S., et al 2013); [1]There are approximately 2,500 new diagnosed case per year in the USA and the incidence of brain tumors has increased slightly over the recent decades which is possibly ascribed to improved diagnostic imaging technologies (Gill, S. K., Panigrahy et al 2013). [2] Despite the fact that the actual incidence and prevalence worldwide is still remains in accurately measured or detected, with $80 \%$ of patients with malignant brain tumors die from the disease.

At present, brain biopsy represents the reference standard for diagnosing brain mass, although the drawbacks of this invasive technique are well known. It is associated with morbidity and mortality, it has sampling errors, and it is not appropriate for screening, longitudinal monitoring, or evaluating treatment response [3]. Among the currently available imaging modalities, magnetic resonance imaging (MRI) and ${ }^{1} \mathrm{H}$ MR spectroscopy (MRS) are the most reproducible, safe, and accurate imaging techniques that can be used in clinical trials and epidemiologic studies [4]. However, MRS has limited clinical applicability or availability because it requires sophisticated post processing methods, and not every MRI is routinely equipped with MRS capabilities. Recent improvements in MRI can provide the magnetic resonance imaging estimated proton density fat fraction (MRI-PDFF), which is a novel biomarker that has demonstrated robust correlation and equivalency with MRS [5-6].
In addition, MRI-PDFF allows fat mapping of the entire liver, and it can be used with any clinical MRI platform, where as MRS measures fat biochemically in small regions of interest (ROIs). To the best of our knowledge, few studies have used MRS and histology to investigate brain content in patients with BTs [7-8].

\section{Materials and Methods}

The study was performed in Royal Care hospital in Khartoum State 38 patients (20 males, 18 females; age range, 4-80 years) have been examined using MRI scanner (1.5 teslaToshiba Avantage-Japan 2009).

\subsection{MRI protocol}

All the cases were examined in supine position with standard circularly polarized head coil using the following sequences. Axial and Sagittal T1WI (550/8.7 ms) TR/TE spin echo. Coronal T2WI (5000/96 ms) TR/TE spin echo. Axial FALIR (9000/92/ms) TR/TE spin echo. $5 \mathrm{~mm}$ section thickness, 230230 Field of view (FOV) and 256-256 matrix size

All of cases which require contrast administration to confirm tumor diagnosis, intravenous administration of Gadolinium- DTPA, contrast enhanced T1WI in axial, sagittal and coronal planes was performed. About 38 cases with confirmed diagnosis MRS were done using single voxel technique. 


\section{International Journal of Science and Research (IJSR) \\ ISSN (Online): 2319-7064}

Index Copernicus Value (2016): 79.57 | Impact Factor (2015): 6.391

\subsection{MRS protocol}

Two localization methods have been performed, each has a different TE. Data were acquired using Point Resolved Spectroscopy (PRESS) pulse sequence and spectroscopic localization has been performed on post contrast T1WI with automatic shimming. All the MR Spectroscopy was performed using single voxel technique initially post contrast imaging was done to localize the tumors and then voxel was placed on volume of interest.

Measurement parameters used in 2D-MRSI were TR/TE: 1500/135 ms, (FOV): 120 _ $120 \mathrm{~mm}$, section thickness: 10 $\mathrm{mm}$ and total scan time was $7 \mathrm{~min}$. The Region of interest (ROI) was carefully placed to avoid strong interference from subcutaneous fat and lipids of the skull, and outer volume suppression (OVS) slabs outside the ROI was used to further reduce the potential for artifact. Measurement parameters used in SVS scans were 1500/35 ms (TR/TE) and voxel size was about $1.5 \mathrm{~cm} 3$. The total Scan time was $3.14 \mathrm{~min}$. 1.2

Analysis of the spectroscopic data: The main metabolites identified by MRS are (NAA) at $2.02 \mathrm{ppm},(\mathrm{Cr})$ at $3.0 \mathrm{ppm}$ and (Cho) at $3.2 \mathrm{ppm}$. Concerning lipids and lactate (LL) were qualitatively defined and estimated their sum between 0.9 and 1.3 parts per mil (ppm). The following metabolite ratio was calculated using the standard commercial software. A spectrum was excluded for analysis if integration of any peak could not be accomplished using the automated analysis software.

$36700-70300+8400$

\begin{tabular}{|c|c|c|c|c|}
\hline Metabolite & \begin{tabular}{c|} 
Major \\
resonance $(\mathrm{ppm})$ \\
\end{tabular} & Physiological significant & Increase & Decrease \\
\hline NAA & 2.0 & $\begin{array}{c}\text { Marker of neuronal health. See only in } \\
\text { neuronal tissues }\end{array}$ & Rarely in canavan disease & Neural damage \\
\hline Cho & 3.2 & $\begin{array}{c}\text { Marker of membrane synthesis and NO } \\
\text { of cells }\end{array}$ & Active tumors growth & $\begin{array}{c}\text { HIV \& Liver } \\
\text { disease }\end{array}$ \\
\hline $\mathrm{Cr}$ & 3.0 & $\begin{array}{c}\text { Energy metabolism. stable in many } \\
\text { disease }\end{array}$ & Trauma & Tumors \&hypoxia \\
\hline Lipids (Lip) & $0.8-1.4$ & Normally not present or very low & Necrotic tumors ,stroke and abscess & - \\
\hline Lactate (Lac) & 1.3 & Normally not present or very low & Necrotic tumors ,stroke and abscess & - \\
\hline $\begin{array}{c}\text { Glutamate \& } \\
\text { Glutamine (Glx) }\end{array}$ & $2.1-2.6$ & Regulation of neurotransmitter activity & $\begin{array}{l}\text { Hepatic encephalopathy\& severe } \\
\text { hypoxia }\end{array}$ & - \\
\hline Myo-inositol (MI) & 3.5 & Used as astrocytes marker & Glioma, Alzheimer and gliomatosis & $\begin{array}{c}\text { Hepatic } \\
\text { encephalopathy }\end{array}$ \\
\hline
\end{tabular}

MRS (single or multi-voxel technique) is noninvasive diagnostic procedure for brain metabolite that could register the pattern of tissue with chemical compounds (Choline compounds (Cho), Creatine and Phosphocreatine (Cr), NAcetyl-Aspartate (NAA), and Lactate (Lac)) and map out the spatial distribution of metabolites within the brain.

The diagnostic findings of MRS depend on the following: (High Choline indicate brain malignancy, weak NAA signal refers to replacement of healthy brain tissue by tumor cells, level of $\mathrm{Cr}$ refers to high energy metabolism and Lac refers to anaerobic metabolism).

\section{Results}

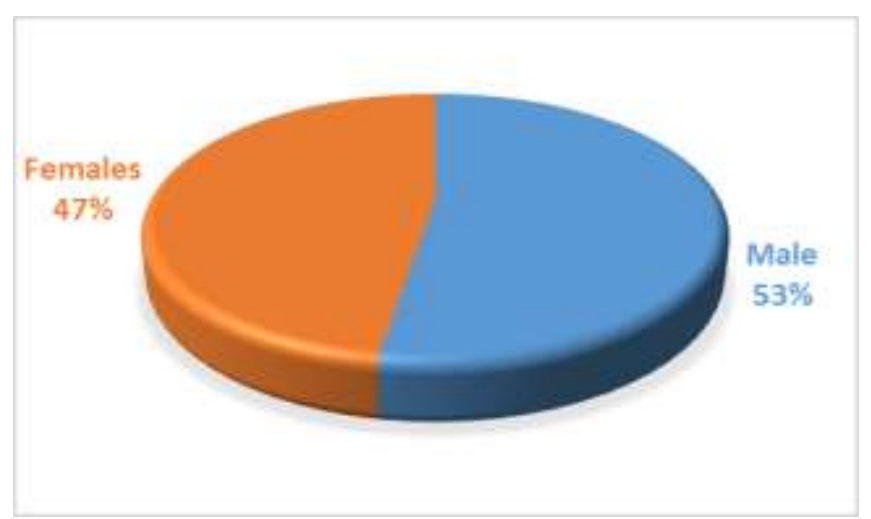

Figure 1: Shows the gender distribution in this study.
Table 1: Demonstrate the correlation of histopathology with Magnetic Resonance Spectroscopy of human biopsies

\begin{tabular}{|c|c|c|c|}
\hline $\mathrm{No}$ & MRS Result & Histopathology Result & \\
\hline 1. & Malignant & Malignant & \\
\hline 2. & Malignant & Malignant & \\
\hline 3. & Malignant & Malignant & \\
\hline 4. & Malignant & Malignant & \\
\hline 5. & Benign & Benign & \\
\hline 6. & Malignant & Malignant & \\
\hline 7. & Malignant & Malignant & \\
\hline 8. & Cystic & Benign & $*$ \\
\hline 9. & Malignant & Malignant & \\
\hline 10. & Malignant & Malignant & \\
\hline 11. & Malignant & Malignant & \\
\hline 12. & Benign & Benign & \\
\hline 13. & Neoplastic tumor & Necrotic tumor & $*$ \\
\hline 14. & Malignant & Malignant & \\
\hline 15. & Abscess & Abscess & \\
\hline 16. & Benign & Malignant & $*$ \\
\hline 17. & Malignant & Malignant & \\
\hline 18. & Malignant & Malignant & \\
\hline 19. & Malignant & Malignant & \\
\hline 20. & Malignant & Benign & $*$ \\
\hline 21. & Malignant & Malignant & \\
\hline 22. & Benign & Benign & \\
\hline 23. & Malignant & Malignant & \\
\hline 24. & Malignant & Malignant & \\
\hline 25. & Benign & Benign & \\
\hline 26. & Benign & Benign & \\
\hline 27. & Benign & Benign & \\
\hline 28. & Benign & Benign & \\
\hline 29. & Malignant & Malignant & \\
\hline
\end{tabular}


International Journal of Science and Research (IJSR)

ISSN (Online): 2319-7064

Index Copernicus Value (2016): 79.57 | Impact Factor (2015): 6.391

\begin{tabular}{|c|c|c|c|}
\hline 30. & Malignant & Malignant & \\
\hline 31. & Malignant & Malignant & \\
\hline 32. & Malignant & Malignant & \\
\hline 33. & Inflammation & Inflammatory & \\
\hline 34. & Malignant & Malignant & \\
\hline 35. & Malignant & Malignant & \\
\hline 36. & Malignant & Benign & $*$ \\
\hline 37. & Malignant & Malignant & \\
\hline 38. & Benign & Benign & \\
\hline
\end{tabular}

Our results suggest that MRS is an accurate, noninvasive diagnostic technique for quantifying brain tumors.

\section{References}

[1] De Moor, J. S., Mariotto, A. B., Parry, C., Alfano, C. M., Padgett, L., Kent, E. E., ... \& Rowland, J. H. (2013). Cancer survivors in the United States: prevalence across the survivorship trajectory and implications for care. Cancer Epidemiology and Prevention Biomarkers, 22(4), 561-570.

Note (*) in table referred to deference between the MRS results and histopathology results.

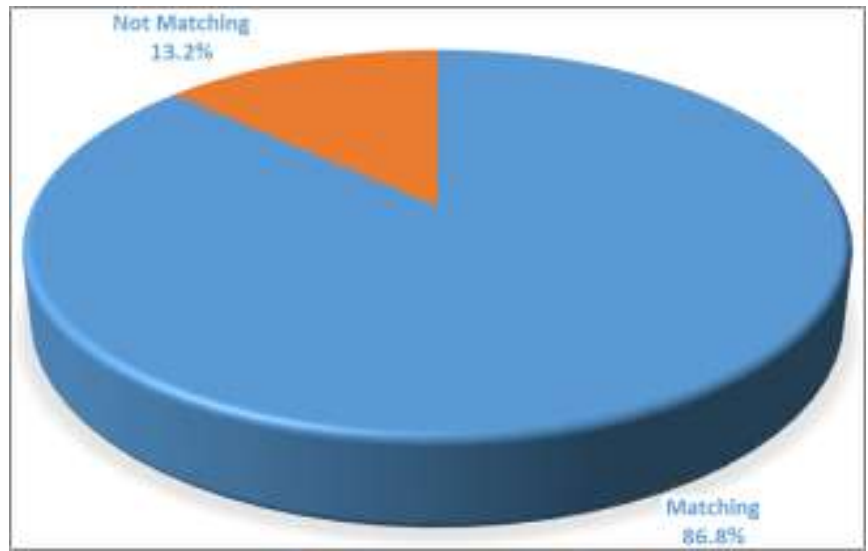

Figure 2 shows the percentage of results matching between the MRS and Histopathology results.

The basis of the MRS method for identifying tumor pathology is the increase in MR visible chemical species with the onset and the development of the disease. Cancer of the uterine cervix was chosen for the first clinical 1H MRS study as, histologically, the distinction between the presence and the absence of malignancy is made with a high level of accuracy.

Our study include 38 brain tumor patients who referred for magnetic spectroscopy MRS at Radiology department at Royal Care hospital (RCH), Radiation and Isotopes Center of Khartoum (RICK) and Alshaab Hospitals in Sudan during 2014-2017.

Out of the patients, $53 \%$ (20) patients were males and $47 \%$ (18) patients were female (figure 1).

The diagnostic study of BTs in Khartoum state using MRS showed little variation in view of accuracy relative to histological findings and our results similar to the Gold standard result; as in Fig. (2); in which MRS showed excellent diagnostic achievement relative to standard (histology) with accuracy $86.8 \%$.

\section{Conclusion}

The magnetic resonance spectroscopy has a role for evaluating patients with brain tumors by interpretations the MR spectroscopy findings for the specific diagnosis. The study showed that male patients are more than female patients, the most common brain pathologies diagnosed by MR spectroscopy is malignancy followed by benign.

[2] Gill, S. K., Panigrahy, A., Arvanitis, T. N., \& Peet, A. C. (2013). Magnetic resonance spectroscopy of pediatric brain tumors. In MR Spectroscopy of Pediatric Brain Disorders (pp. 45-60). Springer New York.

[3] Loomba R, Sirlin CB, Schwimmer JB, Lavine JE. Advances in pediatric nonalcoholic fatty liver disease. Hepatology 2009; 50: 1282-1293 [PMID: 19637286 DOI: $10.1002 /$ hep.23119]

[4] Kotronen A, Yki-Järvinen H. Fatty liver: a novel component of the metabolic syndrome. Arterioscler Thromb Vasc Biol 2008; 28: 27-38 [PMID: 17690317 DOI: 10.1161/ATVBAHA.107.147538]

[5] Noureddin M, Lam J, Peterson MR, Middleton M, Hamilton G, Le TA, Bettencourt R, Changchien C, Brenner DA, Sirlin C, Loomba R. Utility of magnetic resonance imaging versus histology for quantifying changes in liver fat in nonalcoholic fatty liver disease trials. Hepatology 2013; 58: 1930-1940 [PMID: 23696515 DOI: 10.1002/hep.26455]

[6] Hines CD, Frydrychowicz A, Hamilton G, Tudorascu DL, Vigen KK, Yu H, McKenzie CA, Sirlin CB, Brittain JH, Reeder SB. T(1) independent, T(2) $(*)$ corrected chemical shift based fat-water separation with multi-peak fat spectral modeling is an accurate and precise measure of hepatic steatosis. J Magn Reson Imaging 2011; 33: 873-881 [PMID: 21448952 DOI: 10.1002/jmri.22514]

[7] Hamilton G, Yokoo T, Bydder M, Cruite I, Schroeder ME, Sirlin CB, Middleton MS. In vivo characterization of the liver fat ${ }^{1} \mathrm{H}$ MR spectrum. NMR Biomed 2011; 24: $784-790$ [PMID: 21834002 DOI: $10.1002 / \mathrm{nbm} .1622]$

[8] Tang A, Tan J, Sun M, Hamilton G, Bydder M, Wolfson T, Gamst AC, Middleton M, Brunt EM, Loomba R, Lavine JE, Schwimmer JB, Sirlin CB. Nonalcoholic fatty liver disease: MR imaging of liver proton density fat fraction to assess hepatic steatosis. Radiology 2013; 267: 422-431 [PMID: 23382291 DOI: $10.1148 /$ radiol.12120896]. 